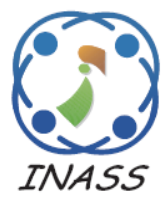

\title{
Automatic Segmentation of Mandibular Cortical Bone on Cone-Beam CT Images Based on Histogram Thresholding and Polynomial Fitting
}

\author{
Rarasmaya Indraswari $^{1 *} \quad$ Agus Zainal Arifin $^{1} \quad$ Nanik Suciati $^{1} \quad$ Eha Renwi Astuti ${ }^{2} \quad$ Takio Kurita $^{3}$ \\ ${ }^{I}$ Department of Informatics, Faculty of Information and Communication Technology, \\ Institut Teknologi Sepuluh Nopember (ITS), Indonesia \\ ${ }^{2}$ Department of Dentomaxillofacial Radiology, Faculty of Dental Medicine, Universitas Airlangga, Indonesia \\ ${ }^{3}$ Department of Information Engineering, Graduate School of Engineering, Hiroshima University, Japan \\ * Corresponding author's Email: rarasmaya16@mhs.if.its.ac.id
}

\begin{abstract}
Automatic segmentation of mandibular cortical bone is challenging due to the appearance of teeth that have similar intensity with the bone tissue and the variety of bone intensity. In this paper we propose a new method for automatic segmentation of mandibular cortical bone on cone-beam computed tomography (CBCT) images. The bone tissue is segmented by using Gaussian mixture model for histogram thresholding. The mandibular inferior cortical bone is obtained by incorporating several polynomial models to fit the structure of cortical bone on coronal slices. The buccal and lingual cortical plate is separated by using histogram thresholding for teeth elimination and polynomial fitting for shape extraction. After performing 3D reconstruction, the volumetric cortical bone is obtained. The proposed method gives average accuracy, sensitivity, and specificity value of $96.82 \%, 85.96 \%, 97.60 \%$, respectively. This shows that the proposed method is promising for automatic and accurate segmentation of mandibular cortical bone on CBCT images.
\end{abstract}

Keywords: Cone-beam computed tomography, Mandibular cortical bone, Histogram thresholding, Polynomial fitting, Segmentation.

\section{Introduction}

Cone-beam computed tomography (CBCT) has been used for wider applications in dentistry due to some limitations of conventional CT [1]. CBCT provides volumetric or three-dimensional (3D) reconstruction of the dento-maxillofacial area. It gives CBCT advantages of other two-dimensional (2D) imaging techniques of dento-maxillofacial area, including panoramic radiographs which is commonly used in dental imaging. Many examination that usually done using the aid of dental panoramic radiographs has been redirected to CBCT because the $3 \mathrm{D}$ reconstruction $\mathrm{CBCT}$ provides more details to help the examiner do the required analysis. Recently, CBCT imaging usually has been used for implant surgery, treatment planning, orthodontic diagnoses, and dental forensics [2].
The measurement of mandibular cortical bone thickness in dental panoramic images has been used for the analysis of bone quality and it has been developed for osteoporosis detection [3-5]. Meanwhile, the analysis of bone quality and quantity in CBCT image is of major importance for the success of implant placement. The amount of cortical bone is responsible for the primary stability of the implant [6]. Therefore, accurate segmentation of mandibular cortical bone becomes a necessity. However, 3D image data such as CBCT generated large amount of 2D slices. The segmentation object in each slice have different position, intensity, and morphology. The large amount of slices in CBCT image presents a problem for manual and semiautomatic segmentation approaches. Therefore, a method to segment the mandibular cortical bone automatically is needed. 
Thresholding based on grayscale intensity is a commonly used method for segmentation of bone tissues on CBCT images [7, 8]. Loubele, et al. (2006) models the grayscale histogram of the selected region of interest (ROI) into a mixture of Gaussian distribution to obtain the threshold to segment bone tissues in multi-slice spiral computed tomography (MSCT) and CBCT images [9]. This approach has been utilized by Hassan, et al. (2010) on his research about influence of scanning and reconstruction on quality of 3D surface model on CBCT [10]. Barandiaran, et al. (2009) use multi-thresholding method by Otsu to separate the input CBCT images into three classes, one of them representing the mandibular structure [11]. However, the resulting segmented images contains not only the bone but also the teeth element.

Nackaerts, et al. (2013) compares the use of global thresholding, which threshold is set manually, and adaptive thresholding for segmentation of trabecular jaw bone on CBCT images [12]. The result shows that adaptive thresholding, that calculates the average intensity around each voxels, generally can produces smaller errors in morphometric indices, while manual threshold selection results in smaller errors in trabecular thickness. Van Eijnatten, et al. (2016) assess the impact of manual threshold selection for medical additive manufacturing of skull model from CT images [7]. This research demonstrates that human factor influences the selection of manual threshold and that no single bone threshold value is applicable for all facial bones. Cuadros Linares, et al. (2018) use a manual selected threshold value to filter the bone and teeth structures on CBCT image [13]. This research aims to separate the mandible with the maxilla and reconstruct the $3 \mathrm{D}$ image.

Segmentation of jaw bone tissues on CBCT images is challenging due to the appearance of teeth that usually have similar intensity with the bone tissues [14]. Moreover, intensity of bone tissues varies and using grayscale thresholding to separates the bone tissues with other elements of CBCT will gives a complete jaw bone tissues, not only the mandibular cortical bone, as the segmentation object [15]. To solve this problem, methods which incorporate prior information about the expected shapes and position of the segmentation objects have been proposed.

Statistical shape model (SSM), which use samples of the object's shape in the training set for generating the statistical model of the object, has been used for segmentation of bone tissues on CT images [16-18]. Abdolali, et al. (2017) proposed to segment mandibular canal in CBCT using conditional SSM and fast marching algorithm. Using $120 \mathrm{CBCT}$ data as the training and test set, the conditional SSM gives the average Dice's coefficient and average symmetric surface distance (ASSD) as 0.9138 and $0.7071 \mathrm{~mm}$, respectively, for segmentation of mandibular bone [18]. Although SSM-based methods can solve the drawbacks of thresholding method, it need a good quality and high number of training datasets that affect the generalization ability and specificity of the method [8]. Other approaches such as using atlas-based registration [2] and machine learning are also facing the same problem [15]. Providing data in large quantities for the training set will be difficult, therefore another automatic method that does not need training data and consider the prior information about the object's shape is needed.

In this paper we propose a new method for automatic segmentation of mandibular cortical bone on CBCT images based on histogram thresholding and polynomial fitting. This method can segment the mandibular cortical bone into several parts automatically without the aid of training set. We extract the mandibular inferior cortical bone, buccal mandibular cortical plate, and lingual mandibular cortical plate from the coronal and axial slices of the CBCT image. The segmentation of mandibular cortical bone on CBCT slices are done automatically by using the global intensity of the image and prior information about the shape of cortical bone, which is similar to a parabola. The grayscale intensity that gives best separation of the bone element from other elements on CBCT images, such as teeth, is obtained automatically and adaptively, depending on each CBCT images. The obtained volumetric cortical bone can be used for measuring the bone quality and quantity. This further can be developed to aid the process of implant placement and bone mineral density measurement.

This paper is organized as follows. Section 2 presents the CBCT dataset that was used for the experiments and the proposed methodology for segmentation of mandibular cortical bone on CBCT images. Section 3 describes the experiments that have been performed, such as the determination of parameter value and comparison with existing methods, and presents its results. The discussion of the experimental results with regards to the existing researches is explained in section 4 . The conclusion and the future work of this research are presented in section 5 . 


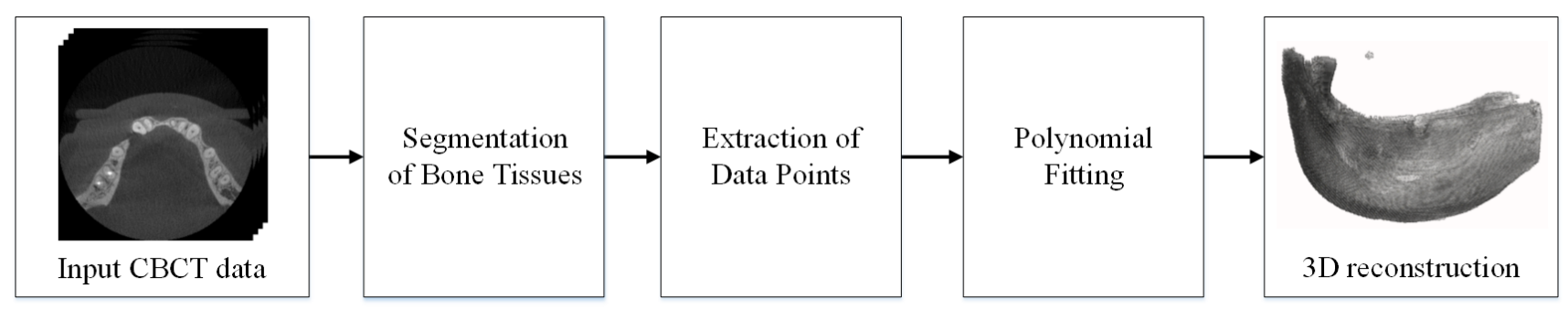

Figure. 1 Flowchart of the proposed method

\section{Material and Methods}

The proposed method consists of four main steps, which are the segmentation of bone tissues, extraction of data points, polynomial fitting, and 3D reconstruction. The flowchart of the proposed method is shown in Fig. 1. The segmentation of mandibular inferior cortical bone is performed on the coronal slices of CBCT image. The separation of buccal and axial cortical plate is performed on the axial slices. The segmentation process is done by incorporating histogram thresholding to obtain the jaw bone tissues and polynomial fitting to extract the shape of cortical bone. The segmentation results of the $2 \mathrm{D}$ slices is combined into $3 \mathrm{D}$ matrix and after performing $3 \mathrm{D}$ reconstruction the volumetric cortical bone is obtained.

\subsection{CBCT dataset}

The dataset that has been used in this research is CBCT scan of human's jaw. CBCT is a medical imaging technique that produces sequence of $2 \mathrm{D}$ images that can be reconstructed into a 3D image. During image acquisition process, the CBCT scanner rotates around the patient's head. According to the rotation of the scanner device, CBCT can be visualized using multiplanar reformation as coronal (anterior to posterior), sagittal (left to right), and axial (maxilla to mandibular) view. Fig. 2 shows the example of 3D CBCT image. Its visualization using multiplanar reformation is shown in Fig. 3. In general, CBCT image contains up to 4 elements, which are air, soft tissues, bone, and tooth [9]. The air and soft tissues element have low intensity while the bone and tooth element have high intensity on the grayscale histogram.

We acquired the CBCT scan from the hospital Rumah Sakit Gigi dan Mulut, Universitas Airlangga (RSGM UNAIR), which used an ORTHOPANTOMOGRAPHTM OP300 3D X-ray unit. The field of view (FOV) width and height of the scanner are $79.8 \mathrm{~mm}$ and $60 \mathrm{~mm}$, respectively. The dataset consisted of jaw images from 8 patients. The segmentation object was mandibular cortical bone,

specifically the inferior cortical bone, buccal cortical plate, and lingual cortical plate as shown in Fig. 2. Inferior cortical bone is located at the bottom part of the jaw. Buccal cortical plate is located on the outer side of the jaw and near the face while lingual cortical plate is located on the inner side of the jaw and near the tongue. The manually annotated ground truth was confirmed by dental radiologist experts. Each data had sizes of $266 \times 266 \times 200$ voxels. The data is sliced according to axial plane, hence for each $3 \mathrm{D}$ data there is 200 axial slices with size $266 \times 266$ pixels.
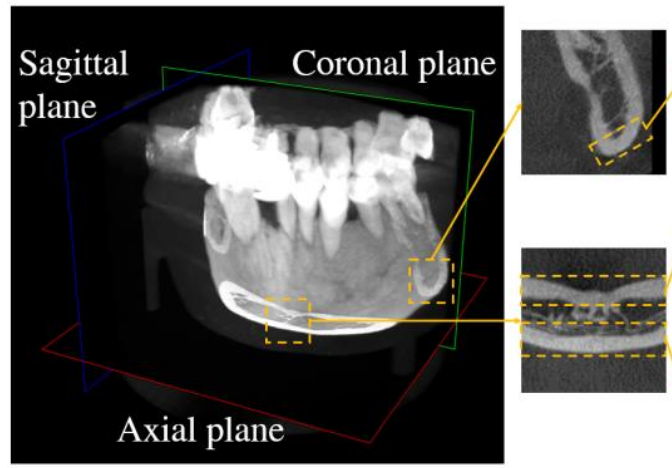

Inferior cortical bone

Lingual cortical plate

Buccal cortical plate

Figure. 2 Volumetric CBCT image

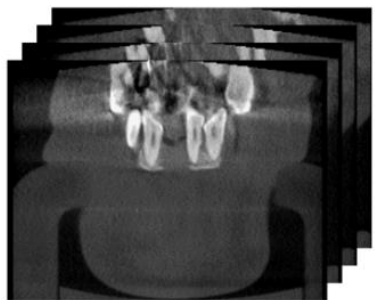

(a)

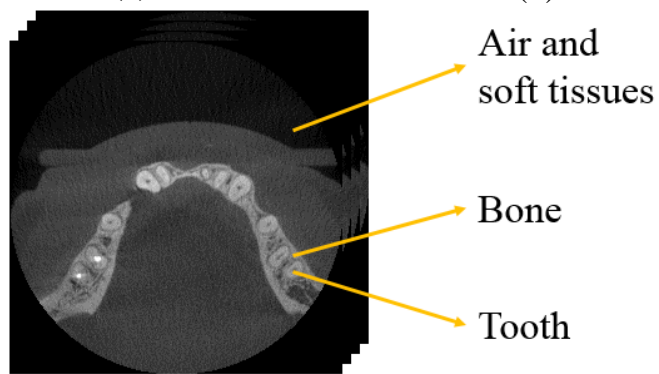

(c)

Figure. 3 Multiplanar reformation: (a) coronal slices, (b) sagittal slices, and (c) axial slices 


\subsection{Segmentation of bone tissues}

There are $n$ slices of CBCT image in axial view with DICOM (Digital Imaging and Communications in Medicine) file format. The depth of CBCT image is depend on the scanner. In case of the depth that is more than 8-bit (0-255 gray level), the CBCT image is converted into 8-bit image. Let $\max$ and $\min$ be the maximal and minimal intensity in all the $n$ CBCT slices, respectively. The new 8-bit value $x^{\prime}$ of the original pixel's value $x$ can be calculated using Eq. (1). Besides converting the CBCT image into 8-bit, this formula also stretch the contrast of the image, therefore it will be easier to process. This enhanced 8-bit CBCT slices is the one that will be processed further.

$$
x^{\prime}=\frac{x-\min }{\max -\min } \times 255
$$

The first step in the cortical bone segmentation process is segmentation of jaw bone that separates the bone element with other elements such as air and soft tissues. The jaw bone segmentation is done using histogram thresholding. This process is done to all of the axial and coronal slices. In general, CBCT image contains up to 4 elements, which are air, soft tissues, bone, and tooth that formed mixture of Gaussian distributions on histogram [9], as shown in Fig. 4. On Fig. 4, the first and second mode represents the air and soft tissues element, while the last mode represents the bone and tooth element. To separate the bone and tooth element from other elements, a threshold between the second and third mode must be determined. Gaussian Mixture Model (GMM) can be used to obtain the thresholds between each mode.

Let $k$ be the number of Gaussian distributions in the data. For each gray level in the histogram, the responsibility of each Gaussian component is calculated using Expectation-Maximization (EM) algorithm. Using the responsibility value, the mean and standard deviation of each Gaussian distribution will be updated to maximize the likelihood of the gray levels assigned in each Gaussian component. This process is repeated until it converges to local optimum. The result is $k$ Gaussian distributions with $(k-1)$ thresholds $\left(t_{1}, t_{2}, \ldots, t_{k-1}\right)$ that separates each of the Gaussian component.

In this research, we assign $k=4$ according on the number of elements in CBCT image. The gray level that become the boundary of the second and third Gaussian component $\left(t_{2}\right)$ is then selected as the bone threshold. For separating the bone and tooth element from the other elements, intensities that are

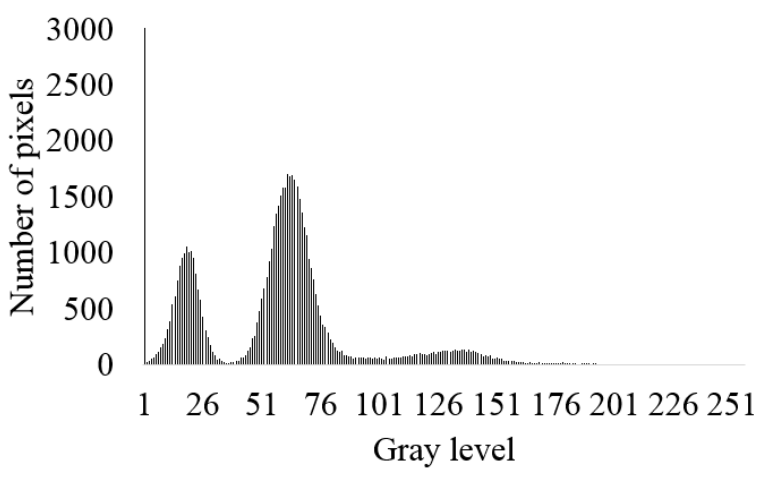

Figure. 4 Grayscale intensity histogram of a CBCT slice

lower than $t_{2}$ are considered as the background while the remaining intensities are considered as the object

\subsection{Extraction of inferior cortical bone}

An approach based on second order polynomial curve fitting is used to separate the inferior cortical bone with the other part of jaw bone because its shape is similar with quadratic function. Separating lines is drawn in the coronal slices of CBCT image according to the polynomial function to separates the area of inferior cortical bone with the others. The area above the separating line will be preserved while the area below the separating line will be eliminated. The input coronal slices is obtained by slicing the $3 \mathrm{D}$ matrix of CBCT image $I(x, y, z)$ according to the $x$ axis. The result is 266 coronal slices with size $266 \mathrm{x}$ 200 pixels, as shown in Fig. 5 (a). The input slices is segmented by using the threshold $t_{2}$ that was obtained by GMM method to obtain the bone and teeth element, as shown in Fig. 5 (b). Scanning process is then performed from the bottom to the top of the image to obtain the upper border of inferior cortical bone, which then called as data points. For each column in the image, a data point is defined as the first pixel that has higher intensity than the pixel above it. The example of obtained data points is shown in Fig. 5 (c).

Given a set of data points $P=$ $\left\{\left(x_{1}, y_{1}\right),\left(x_{2}, y_{2}\right), \ldots,\left(x_{M}, y_{M}\right)\right\}$, with $M$ as the number of data points, and polynomial function $f(x)=y=\sum_{r=0}^{n} a_{r} x^{r}$, with $n$ as the order of the polynomial and $a_{r}$ as the variable parameters [19]. Polynomial fitting search the set of variable parameters $a_{r}$ that gives minimal error when applied to the polynomial function with set of points $P$ as the $x$ and $y$ variable. Let $w\left(x_{i}\right)$ and $w\left(y_{i}\right)$ be the weights of perpendicular distance from the given data points $\left(x_{i}, y_{i}\right)$ to the curve, the error minimization is usually done by using least-square error method (LSE) that minimizes the sum of weighted difference $S$ between the data points and the obtained points that lie on the 


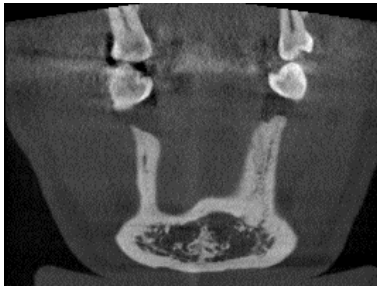

(a)

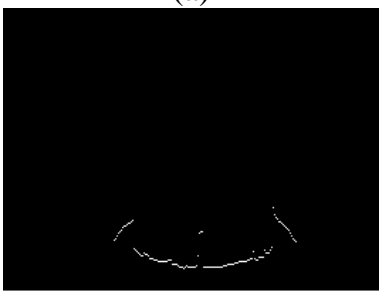

(c)

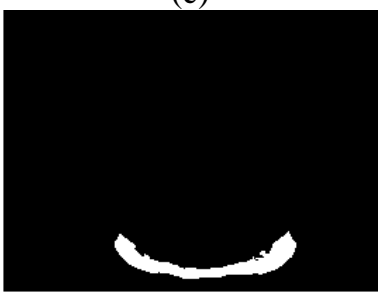

(e)

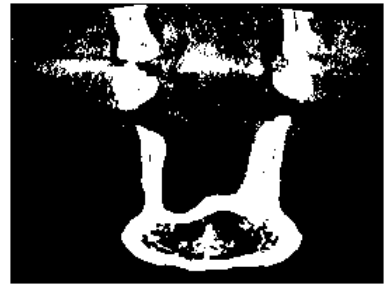

(b)

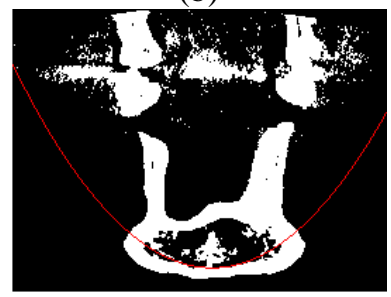

(d)

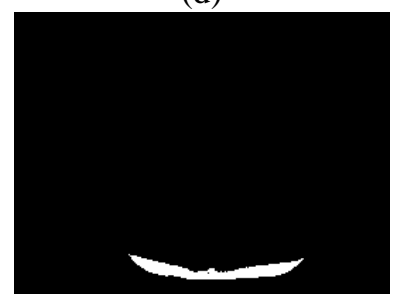

(f)
Figure. 5 Extraction of inferior cortical bone: (a) input coronal slices, (b) the segmented bone and teeth, (c) the extracted data points, (d) the polynomial curve, (e) the obtained inferior cortical bone, and (f) the ground truth

polynomial curve $\left(x_{i}{ }^{\prime}, y_{i}{ }^{\prime}\right)$, as in Eq. (2). In this research we set $w\left(x_{i}\right)=w\left(y_{i}\right)=1$ for all data points $i$, thus gives equal weight for each data points and yields a polynomial curve that is not scale invariant. We assume that both $x_{i}$ and $y_{i}$ is subject to error, therefore the result of $\frac{w\left(x_{i}\right)}{w\left(y_{i}\right)}$ should be a constant value [20]. For the second order polynomial fitting, the number of $n$ is 2 hence the polynomial function of the curve is written as in Eq. (3).

$$
\begin{gathered}
S=\sum_{i=1}^{M}\left\{w\left(x_{i}\right)\left(x_{i}-x_{i}{ }^{\prime}\right)^{2}+w\left(y_{i}\right)\left(y_{i}-y_{i}{ }^{\prime}\right)^{2}\right\} \\
f(x)=a_{0}+a_{1} x+a_{2} x^{2}
\end{gathered}
$$

There is two kind of cortical bone's structures in coronal slices. In the first part of coronal slices the cortical bone appears as one object at the bottom center of the image, while in the last slices the cortical bone appears as two objects at the left and the right side of the image. To recognize whether there are one or two objects in the image, we check whether there is a section of object located around the bottom center of the image and whether there are two objects of almost the same size in the image. If there are two objects in the image, the image will be split into two, namely the left image and right image. Then a separating line will be drawn on each image. The

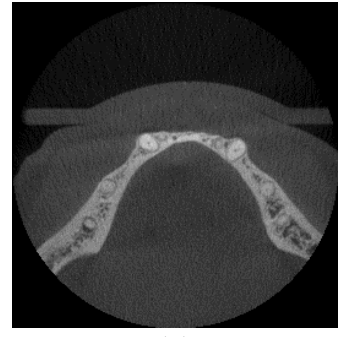

(a)

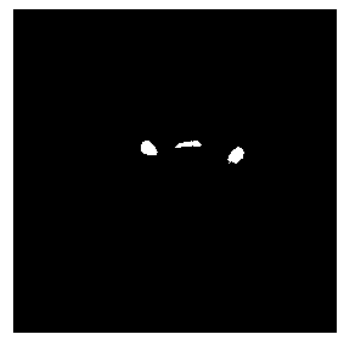

(c)

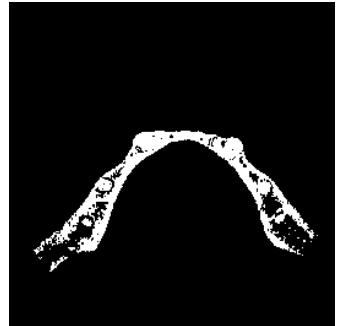

(b)

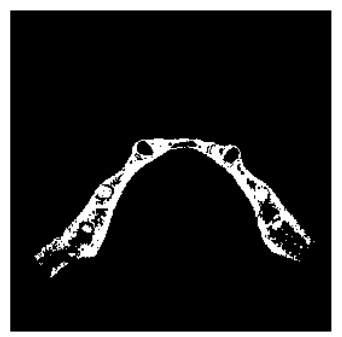

(d)
Figure. 6 Bone segmentation process: (a) input axial slices, (b) the segmented bone and teeth, (c) the segmented teeth, and (d) the obtained bone tissues

example of separating line for one object is shown in Fig. 5 (d). The obtained inferior cortical bone and its ground truth is shown in Fig. 5 (e) and Fig. 5 (f), respectively.

\subsection{Separation of buccal and lingual cortical plate}

For separation of buccal and lingual cortical plate, we use axial slices, as shown in Fig. 6 (a). First, segmentation using threshold $t_{2}$ that was obtained by GMM method is performed to segment the bone and teeth element, which result is shown in Fig. 6 (b). To obtain the teeth element, the boundary of the third and fourth Gaussian component $\left(t_{3}\right)$ is selected as the teeth threshold. The result of teeth segmentation using GMM is shown in Fig. 6 (c). The bone element is then obtained by subtract the bone and teeth image with the teeth image. The result of the bone segmentation on axial slice is shown in Fig. 6 (d).

To separate the buccal and lingual cortical plate, polynomial fitting method is utilized. In axial slices, the buccal cortical plate is located at the top of the lingual cortical plate. To obtain the buccal cortical plate, data points $P$ consist of points at the bottom edge of the buccal cortical plate must be obtained. Looping through all the column from the left to the right side of the object (cortical bone), scanning process is done from the top to the bottom of the image to obtain the data point at each column. A data point is defined as the first pixel that has higher intensity than the pixel below it. Using the obtained set of data points, polynomial fitting is used to drawn a separating line on the bottom edge of the buccal 


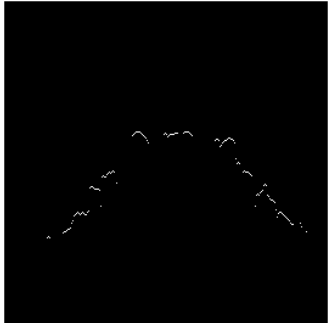

(a)

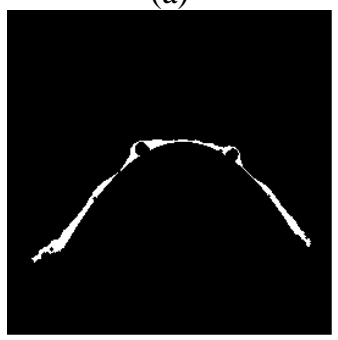

(c)

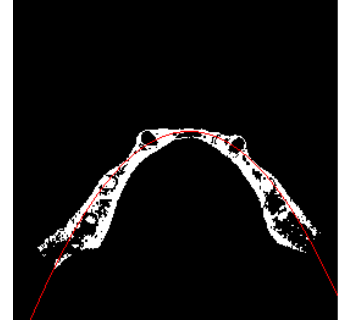

(b)

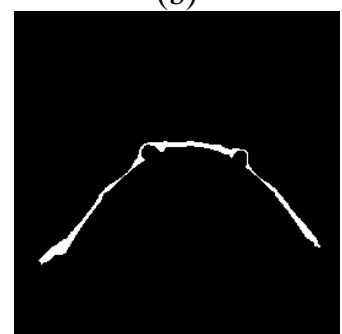

(d)
Figure. 7 Extraction of buccal cortical plate: (a) the obtained data points, (b) the polynomial curve, (c) the obtained buccal cortical plate, and (d) the ground truth

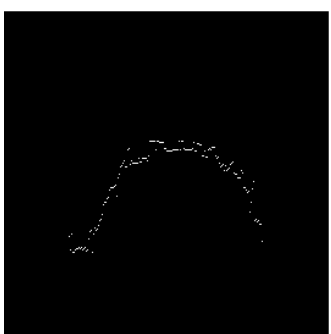

(a)

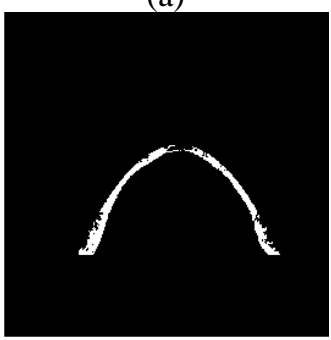

(c)

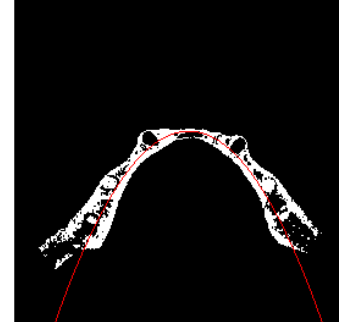

(b)

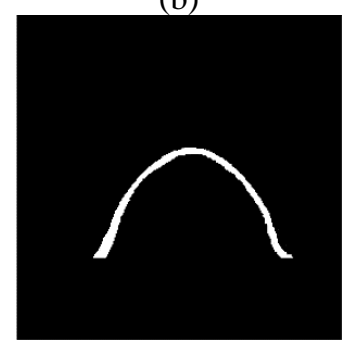

(d)
Figure. 8 Extraction of lingual cortical plate: (a) the obtained data points, (b) the polynomial curve, (c) the obtained lingual cortical plate, and (d) the ground truth

cortical plate to separate it from other part of the jaw bone. Area above the separating line will be preserved while area below the separating line will be eliminated. The process of extracting buccal cortical plate is shown in Fig. 7.

To obtain the lingual cortical plate, by using the bone segmentation result, a separating line will be drawn at the upper edge of the lingual cortical plate. Scanning process is done from the bottom to the top of the image to obtain the data point, which is defined as the first pixel that has higher intensity than the pixel above it. Polynomial fitting is performed to obtain the separating line. Area below the separating

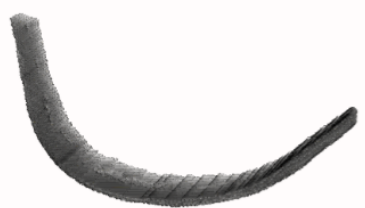

(a)

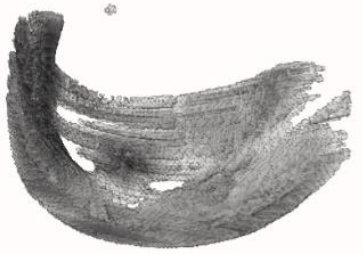

(c)

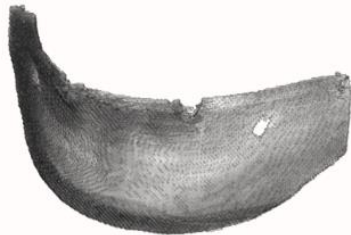

(b)

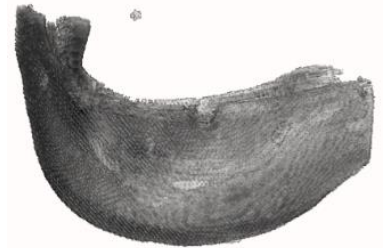

(d)
Figure. 9 The 3D reconstruction of (a) mandibular inferior cortical bone, (b) mandibular buccal cortical plate, (c) mandibular lingual cortical plate, and (d) mandibular cortical bone

line will be preserved while area above the separating line will be eliminated. The process of extracting lingual cortical plate is shown in Fig. 8.

\subsection{The 3D reconstruction}

CBCT image construct a 3-dimensional matrix $I(x, y, z)$ with size $l \times m \times n$ pixels where separation based on the $x$-axis will generate $l$ coronal slices with size $m \times n$ pixels, separation based on the $y$-axis will generate $m$ sagittal slices with size $l \times n$ pixels, and separation based on the $z$-axis will generate $n$ axial slices with size $l \times m$ pixels. The 3D reconstruction of the coronal slices in Section 2.2 based on the $x$-axis will result in the segmented mandibular inferior cortical bone. The 3D reconstruction of the axial slices in Section 2.3 based on the $z$-axis will result in the segmented mandibular buccal and lingual cortical plate, separately. To obtain a complete mandibular cortical bone, we can combine the segmentation result of buccal and lingual cortical plate. This will eliminate the trabecular bone from the 3D image. Fig. 9 shows the result of 3D reconstruction of the mandibular inferior cortical bone, buccal cortical plate, lingual cortical plate, and mandibular cortical bone.

\section{Experimental results}

The performance of the proposed method is measured using accuracy (Acc), sensitivity (Sen), and specificity (Spe) evaluation metrics. Confusion matrix that consist of four elements, namely true positive $T P$, true negative $T N$, false positive $F P$, and false negative $F N$, represents the possible overlap pair between the class of output image $C_{o}$ and class 
of target image $C_{t}$ in binary segmentation [21]. Let $m_{i j}$ become the sum of agreement between each pair of pixels $(i, j)$ in the same coordinate where $i \in C_{o}$ Co and $j \in C_{t}$. TP is the sum of agreement between output class $C_{o}=1$ and target class $C_{t}=1\left(m_{11}\right)$, $T N$ is the sum of agreement between output class $C_{o}=0$ and target class $C_{t}=0\left(m_{00}\right), F P$ is the sum of agreement between output class $C_{o}=1$ and target class $C_{t}=0\left(m_{10}\right)$, and $F N$ is the sum of agreement between output class $C_{o}=0$ and target class $C_{t}=1$ $\left(m_{01}\right)$.

Accuracy measures the percentage of the correctly classified pixels on the whole image, which formula is written in Eq. (4). Sensitivity or true positive rate measures the percentage of the correctly classified object pixels, which formula is written in Eq. (5). Specificity or true negative rate measures the percentage of the correctly classified background pixels, which formula is written in Eq. (6). The sensitivity value is not measured in the slices that contain no segmentation object. Segmentation result is considered as good if it gives high accuracy, sensitivity, and specificity value. High sensitivity value means that the method have good ability to detect object class, while high specificity means that the method have good ability to detect background class.

$$
\begin{aligned}
& A c c=\frac{T P+T N}{T P+T N+F P+F N} \\
& \text { Sen }=\frac{T P}{T P+F N} \\
& \text { Spe }=\frac{T N}{T N+F P}
\end{aligned}
$$

\subsection{Comparison of bone tissues segmentation methods}

The performance of the proposed method, that is using Gaussian mixture model for the segmentation of bone tissues, is compared with using Otsu's multithresholding [22] and histogram cluster analysis (HCA) [23] for the segmentation of bone tissues. Tables $1-3$ show the performance comparison for the segmentation of mandibular inferior cortical bone, buccal cortical plate, and lingual cortical plate, respectively. The highest sensitivity value is obtained using GMM as the method for segmenting the bone tissues. The average accuracy, sensitivity, and specificity value of the proposed method using GMM are $96.82 \%, 85.96 \%, 97.60 \%$, respectively. The average accuracy, sensitivity, and specificity value by using Otsu's multi-thresholding are $96.40 \%$, $78.21 \%, 97.30 \%$, respectively. The average accuracy,
Table 1. Comparison of Bone Segmentation Methods for Segmentation of Mandibular Inferior Cortical Bone

\begin{tabular}{|c|c|c|c|}
\hline Method & Acc (\%) & Sen (\%) & Spe (\%) \\
\hline $\begin{array}{c}\text { Gaussian } \\
\text { Mixture Model }\end{array}$ & 92.98 & $\mathbf{8 5 . 2 6}$ & 95.19 \\
\hline $\begin{array}{c}\text { Otsu's Multi- } \\
\text { thresholding }\end{array}$ & 92.83 & 84.68 & 95.27 \\
\hline $\begin{array}{c}\text { Histogram } \\
\text { Cluster } \\
\text { Analysis }\end{array}$ & 93.40 & 67.94 & 97.98 \\
\hline
\end{tabular}

Table 2. Comparison of Bone Segmentation Methods for Segmentation of Mandibular Buccal Cortical Plate

\begin{tabular}{|c|c|c|c|}
\hline Method & Acc (\%) & Sen (\%) & Spe (\%) \\
\hline $\begin{array}{c}\text { Gaussian } \\
\text { Mixture Model }\end{array}$ & 99.00 & $\mathbf{8 7 . 8 1}$ & 99.03 \\
\hline $\begin{array}{c}\text { Otsu's Multi- } \\
\text { thresholding }\end{array}$ & 98.76 & 66.41 & 98.93 \\
\hline $\begin{array}{c}\text { Histogram } \\
\text { Cluster } \\
\text { Analysis }\end{array}$ & 99.60 & 79.26 & 99.69 \\
\hline
\end{tabular}

Table 3. Comparison of Bone Segmentation Methods for Segmentation of Mandibular Lingual Cortical Plate

\begin{tabular}{|c|c|c|c|}
\hline Method & Acc (\%) & Sen (\%) & Spe (\%) \\
\hline $\begin{array}{c}\text { Gaussian } \\
\text { Mixture Model }\end{array}$ & 98.49 & $\mathbf{8 4 . 8 1}$ & 98.57 \\
\hline $\begin{array}{c}\text { Otsu's Multi- } \\
\text { thresholding }\end{array}$ & 97.62 & 83.53 & 97.71 \\
\hline $\begin{array}{c}\text { Histogram } \\
\text { Cluster } \\
\text { Analysis }\end{array}$ & 99.30 & 72.98 & 99.48 \\
\hline
\end{tabular}

sensitivity, and specificity value by using HCA are $97.43 \%, 73.39 \%, 99.05 \%$, respectively.

Otsu's thresholding method select the gray level $k$ in the histogram that gives the maximum inter-class variance $\sigma_{B}^{2}(k)$ according to Eq. (7) as the threshold that gives the best separability between the clusters of image intensity. The separation between cluster 0 (background) and cluster 1 (object) is calculated based on the probability of the cluster occurrence $\left(\omega_{0}\right.$ and $\left.\omega_{1}\right)$ and the average gray level in the cluster $\left(\mu_{0}\right.$ and $\left.\mu_{1}\right)$. Maximizing the inter-class variance is equivalent to minimizing the intra-class variance, which is equivalent to the maximization of the likelihood of the Gaussian distribution with a common variance [24]. In this experiment we use Otsu's method to choose three thresholds that separates the grayscale intensity histogram into four clusters.

$$
\sigma_{B}^{2}(k)=\omega_{0} \omega_{1}\left(\mu_{1}-\mu_{0}\right)^{2}
$$


HCA assume the gray level histogram with nonzero element as cluster. The inter-class variance $\left(\sigma_{I}^{2}\right)$ and intra-class variance $\left(\sigma_{A}^{2}\right)$ of each cluster $\left(C_{k 1}\right.$ and $C_{k 2}$ ) then calculated according to Eq. (8) to determine which cluster to be merged. Hierarchical clustering is then carried out until there are only $k$ number of clusters left. In this experiment, we set the number of final cluster $k$ for the HCA method as four to give an equal comparison with GMM and Otsu's thresholding method that separates the histogram into four clusters. Fig. 12 - Fig. 14 shows the obtained threshold between each Gaussian distributions on the image histogram using GMM, Otsu's multithresholding, and HCA method, respectively.

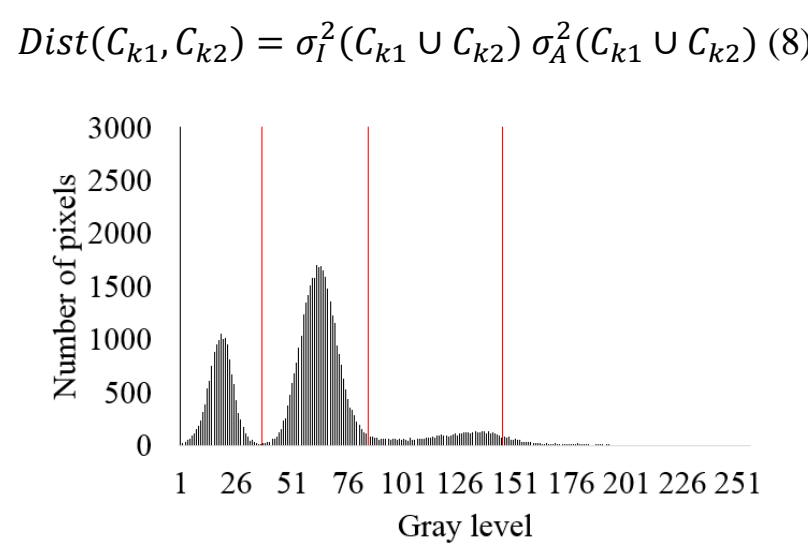

Figure. 10 Image histogram and the obtained Gaussian threshold using GMM with $k=4$

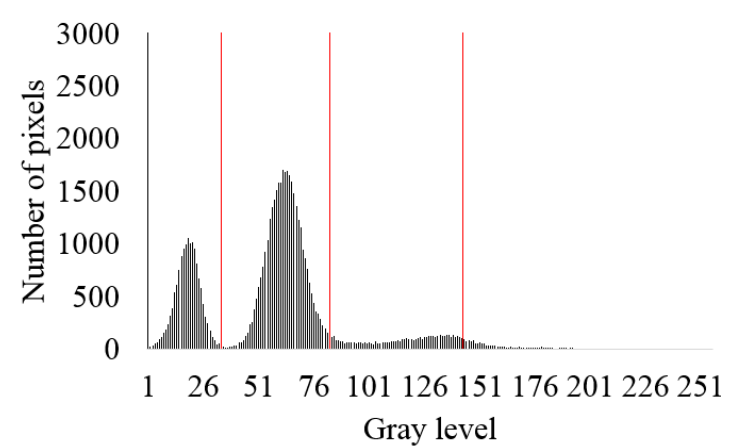

Figure. 11 Image histogram and the obtained Gaussian threshold using Otsu multi-thresholding

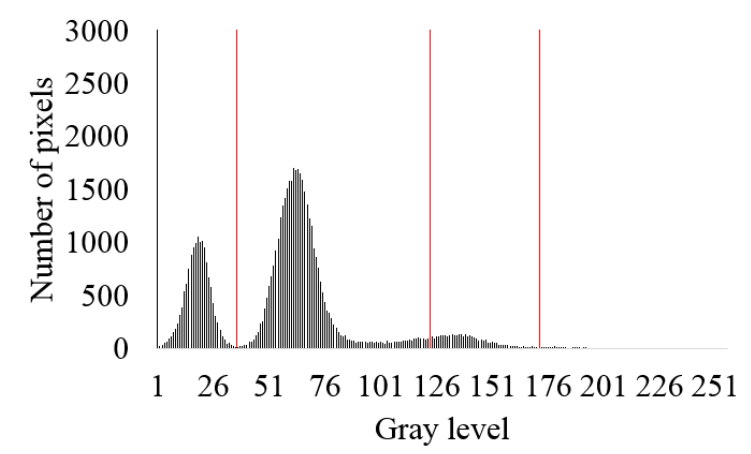

Figure. 12 Image histogram and the obtained Gaussian threshold using HCA
Table 4. Segmentation performance with the different number of Gaussian distribution

\begin{tabular}{|c|c|c|c|}
\hline$k$ & Acc (\%) & Sen $(\%)$ & Spe $(\%)$ \\
\hline 3 & 92.91 & 85.06 & 96.02 \\
\hline 4 & 92.98 & $\mathbf{8 5 . 2 6}$ & 95.19 \\
\hline 5 & 93.13 & 85.13 & 95.32 \\
\hline 6 & 93.10 & 85.22 & 95.06 \\
\hline 7 & 93.48 & 72.06 & 97.50 \\
\hline
\end{tabular}

\subsection{Effect of the number of Gaussian model}

In this experiment we try different value of $k$, a parameter in the Gaussian mixture model that represent the number of Gaussian distribution in the image. The GMM method will resulting in $\left\{t_{i} \mid i=\right.$ $1,2, \ldots, k-1\}$ thresholds. The value of $i$ that was selected for each $k$, in which $t_{i}$ will be used to separate the bone element, is chosen based on our observation of the obtained separation border between each Gaussian distribution on the image histogram. The performance of the proposed method using the selected $k$ value for segmentation of mandibular inferior cortical bone is presented on Table 4 . The highest sensitivity is obtained using four as the number of Gaussian distribution in the image.

\subsection{Image registration for refinement}

We try to add refinement process using image registration in our methodology. The refinement process is added before $3 \mathrm{D}$ reconstruction process. Taking into account that CBCT consisted of sequence of 2-dimensional images, the image on adjacent slices are almost similar. It means that the segmentation result of adjacent slices should not be much different. To improve the segmentation result, refinement process using demon registration as in [25] is done.

In this experiment, registration process is done to the input grayscale CBCT slices to obtain the transformation matrix between each slices. Those transformation matrices are implemented to the resulting binary images from segmentation process, sequentially, to improve the similarity between slices that are obtained from segmentation process. The transformation matrix between each slices is calculated on the grayscale image. By using a binary template image of the middle slice $i$ as the starting reference image, the registration process is moving towards the adjacent upper and bottom slice. The binary segmentation image of slice $i$ act as the reference image while the binary segmentation image of slice $i-l$ for the upper slices and the binary image of slice $i+1$ for the bottom slices act as the target image, iteratively. This process will refine the 


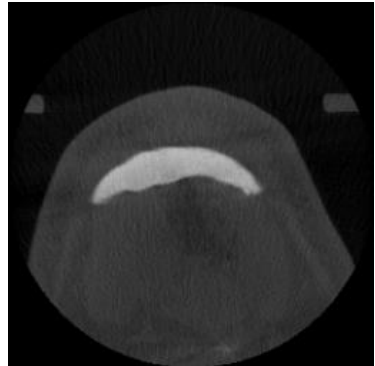

(a)

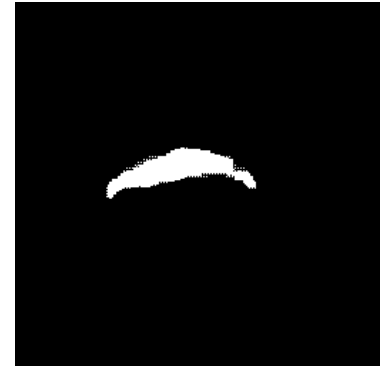

(b)
Figure. 13 (a) Input axial slices and (b) its segmentation result by using U-Net

segmentation result so that the difference of cortical bone shape between adjacent slices is much smaller. The average accuracy, sensitivity, and specificity of the proposed method for segmentation of mandibular lingual cortical bone using image registration for refinement process are $93.21 \%, 89.01 \%$, and $96.71 \%$, respectively.

\subsection{Comparison with deep learning techniques}

In this evaluation, we compared the proposed methodology with U-Net [26], one of deep learning architecture for medical image segmentation. The UNet architecture consists of contracting path (left side) and expansive path (right side). The contracting path consist of repeated convolutions process, each followed by a rectified linear unit (ReLU) and max pooling operation. The expansive path consist of repeated up-convolution process and concatenation with the correspondingly cropped feature map from the contracting path.

The experiment is conducted using training data from 6 patients, in which each patient has 200 images from axial slices, and testing data from 2 patients. Data augmentation is not used for the training process so that it will not disturb the sequence of the 3D data. We set the training epoch as 50 and the batch size as 16. The size of the input image is $256 \times 256$ pixels, according to the size of axial slices on CBCT images. The size of the convolution filter is $3 \times 3$ pixels and the convolution process result in 16 feature maps. The cost function for the network is sigmoid crossentropy because we treat segmentation as classification problem instead of regression. The average accuracy, sensitivity, and specificity value by using U-Net for segmentation of mandibular inferior cortical bone are 99.49\%, 48.52\%, 99.89\%, respectively. The example of input axial slice and its corresponding segmentation result by using U-Net is shown in Fig. 13.

\section{Discussion}

In this research, a method for segmentation of mandibular inferior cortical bone on CBCT images has been presented. The method incorporates prior information of global intensity in CBCT images and cortical bone shape to segment the bone automatically. Thresholding based on the global intensity of the images is the most common approach for detecting elements in CBCT image, such as air, soft tissues, bone, and teeth. This is because CBCT imaging uses Hounsfield Unit (HU) to represent its image intensity values and each of CBCT element has different range value of $\mathrm{HU}$ unit. However, CBCT have no absolute $\mathrm{HU}$ calibration because it varies between scanners and scans [27]. Intensity values of CBCT images are not as uniform or reproducible as the intensity values of other computed tomography imaging technique, such as MSCT images [12]. Human factor on selecting the threshold value may influence the outcome of the segmentation process on CBCT [7]. Therefore, selecting threshold for bone segmentation on CBCT images automatically is a challenging task.

In this paper, Gaussian mixture model with expectation-minimization algorithm is used for segmentation of bone tissues on CBCT image. We choose to select four as the number of Gaussian distribution in the image based on the number of elements in CBCT images. Based on our experiments, separating the image grayscale intensity histogram into four Gaussian distribution also gives the highest sensitivity value. This is in accordance with the research of Loubele, et al. (2006) that said that up to five Gaussian distribution is needed to represent the bone and soft tissue element in CBCT image [9].

We compared the bone segmentation method in the proposed methodology with Otsu's multithresholding [22] and histogram cluster analysis [23]. Those methods search the thresholds in the grayscale intensity histogram that separates the intensity of the CBCT images into four cluster, thus provide an equal comparison with the GMM method. The highest sensitivity value is achieved by using GMM. Higher sensitivity value means that the proposed method has more success in detecting the object, which is cortical bone, than the compared methods. The obtained threshold value by using GMM and Otsu's multithresholding is similar, although the obtained threshold values using Otsu's method have slightly lower grayscale value than the obtained threshold values using GMM. This is because both methods model the intensity histogram into Gaussian distributions. 
Otsu's multi-thresholding is used in [11] for separate the bone element on CBCT image. According to Kurita, et al. (1992) the Otsu's thresholding method is best used on histogram consist of Gaussian distributions with uniform variance [24]. However, since we cannot assume that the Gaussian distributions on the histogram of CBCT image have uniform variance, the obtained thresholds using Otsu's thresholding may be less precise than if the distributions have uniform variance. The obtained thresholds using HCA method tend to give higher value than the obtained thresholds using GMM and Otsu's multi-thresholding. Therefore, by using HCA method, many of the bone tissues are missing and not be segmented properly, resulting in low sensitivity value for the segmentation of inferior cortical bone and lingual cortical plate. Moreover, because CBCT images have much larger background area (non-bone element) than the object area (bone element), the accuracy of HCA method is slightly better than GMM method even though the HCA method is failed to recognize the segmentation object in images.

Image registration has been widely used for medical image analysis because it can determine the corresponding feature between images that collected at different times [28]. It find the best match between two images and find its transformation models. Demon registration is categorized as non-rigid registration technique. It is an approximation to fluid registration, which treat the image as a viscous fluid model that allows large and highly localized deformation [28]. This method is based on pixel velocities, which is caused by edge-based forces, and filter it using Gaussian kernel for global registration [25]. Demon registration gives high precision and computationally efficient compared to other nonrigid registrations [29]. Therefore we choose to integrate it into the proposed method in our experiment. In case of 3D medical images, such as CBCT images, refinement using image registration provides additional information from neighbouring slices. By incorporating the information from neighbouring slices, the incorrect segmentation result of a slice may be refined and the performance of the segmentation method increased. However, because of the image registration method has high computational complexity, using refinement will effect on increment of running time. Therefore, because of the difference of segmentation performance is not very high, using image registration for refinement process is not recommended.

Recent researches have shown that deep learning techniques are useful in medical image analysis, such as image segmentation and computer- aided diagnosis [30, 31]. We compared the proposed methodology with U-Net [26] that provides good and fast performance for segmentation of neuronal structures in electron microscopic stacks and segmentation of cell in light microscopic images. However, the segmentation result using U-Net gives low sensitivity value. Low sensitivity means that the method failed to recognize the inferior cortical bone as the object. However, the U-Net method still gives high accuracy value because the number of pixels that is categorized as the object is relatively small than the number of pixels in the whole image. Therefore, the failure to recognize the object does not have a major effect on the overall accuracy of the method. This support the statement that deep learning techniques required large-scale dataset for the training process to achieve good segmentation result [15, 30-31].

\section{Conclusion}

In this paper we propose a new method for automatic segmentation of mandibular inferior cortical bone on CBCT images. The segmentation is done by incorporating histogram thresholding to obtain the jaw bone tissues and polynomial fitting to extract the shape of cortical bone. The mandibular inferior cortical bone can be separated into buccal and inferior plate according to the axial slices of CBCT image. After performing 3D reconstruction, volumetric cortical bone is obtained. The proposed segmentation method that use Gaussian mixture model with $k=4$ for histogram thresholding gives the average accuracy, sensitivity, and specificity for segmentation of cortical bone of $96.82 \%, 85.96 \%$, $97.60 \%$, respectively.

The high performance of the proposed method shows that it is promising for automatic and accurate segmentation of mandibular cortical bone on CBCT images. The 3D reconstruction of cortical bone can provide specific details of the cortical bone, such as its volume. The separation of buccal and lingual plate of mandibular cortical bone in the proposed method allows for visualization and examination of buccal and lingual cortical plate separately. The obtained volumetric cortical bone is useful for many kind of analysis and applications, such as the measurement of bone density, bone thickness, and bone volume for implant surgery, dental treatment, and orthodontic diagnoses. For future work, since CBCT image is considered as noisy, the use of image enhancement method such as noise reduction should be analyzed to improve the performance of the bone segmentation process. 


\section{Acknowledgments}

This work is supported by the Ministry of Research, Technology, and Higher Education, Indonesia with grant number 128/SP2H/PTNBH/DRPM/2018.

\section{References}

[1] D. A. Tyndall and S. Rathore, "Cone-beam CT diagnostic applications: caries, periodontal bone assessment, and endodontic applications", Dental Clinics of North America, Vol. 52, No. 4, pp. 825-841, 2008.

[2] Y. J. Chuang, B. M. Doherty, N. Adluru, M. K. Chung, and H. K. Vorperian, "A novel registration-based semiautomatic mandible segmentation pipeline using computed tomography images to study mandibular development", Journal of Computer Assisted Tomography, Vol. 42, No. 2, pp. 306-316, 2018.

[3] M. Kavitha, A. Asano, A. Taguchi, T. Kurita, and M. Sanada, "Diagnosis of osteoporosis from dental panoramic radiographs using the support vector machine method in a computeraided system", BMC Medical Imaging, Vol. 12, No. 1, p. 1, 2012.

[4] S. Geary, F. Selvi, S. Chuang, and M. August, "Identifying dental panoramic radiograph features for the screening of low bone mass in postmenopausal women", International Journal of Oral and Maxillofacial Surgery, Vol. 44, No. 3, pp. 395-399, 2015.

[5] R. Indraswari, A. Arifin, N. Suciati, E. Astuti, and S. ITS, "Computer-aided system for osteoporosis assessment using mandibular cortical width measurement on dental panoramic radiographs", In: Proc. of the 21st IADMFR/TAOMFR 2017 World Congress, p. 91, 2017.

[6] T. K. Reddy and N. Kumaravel, "Segmentation and Classification of Jaw Bone CT images using Curvelet based Texture features", Bangladesh Journal of Medical Science, Vol. 9, No. 1, pp. 33-43, 2010.

[7] M. van Eijnatten, J. Koivisto, K. Karhu, T. Forouzanfar, and J. Wolff, "The impact of manual threshold selection in medical additive manufacturing", International Journal of Computer Assisted Radiology and Surgery, Vol. 12, No. 4, pp. 607-615, 2017.

[8] M. van Eijnatten, R. van Dijk, J. Dobbe, G. Streekstra, J. Koivisto, and J. Wolff, "CT image segmentation methods for bone used in medical additive manufacturing", Medical Engineering \& Physics, Vol. 51, pp. 6-16, 2018.

[9] M. Loubele, F. Maes, F. Schutyser, G. Marchal, R. Jacobs, and P. Suetens, "Assessment of bone segmentation quality of cone-beam CT versus multislice spiral CT: a pilot study", Oral Surgery, Oral Medicine, Oral Pathology, Oral Radiology, and Endodontology, Vol. 102, No. 2, pp. 225-234, 2006.

[10] B. Hassan, P. C. Souza, R. Jacobs, S. de Azambuja Berti, and P. van der Stelt, "Influence of scanning and reconstruction parameters on quality of three-dimensional surface models of the dental arches from cone beam computed tomography", Clinical Oral Investigations, Vol. 14, No. 3, pp. 303-310, 2010.

[11] I. Barandiaran, I. Macía, E. Berckmann, D. Wald, M. Dupillier, C. Paloc, and M. Graña, "An automatic segmentation and reconstruction of mandibular structures from CT-data", In: Proc. of International Conference on Intelligent Data Engineering and Automated Learning, pp. 649-655, 2009.

[12] O. Nackaerts, M. Depypere, G. Zhang, B. Vandenberghe, F. Maes, R. Jacobs, and S. Consortium, "Segmentation of trabecular jaw bone on cone beam CT datasets", Clinical Implant Dentistry and Related Research, Vol. 17, No. 6, pp. 1082-1091, 2015.

[13] O. Cuadros Linares, J. Bianchi, D. Raveli, J. Batista Neto, and B. Hamann, "Mandible and skull segmentation in cone beam computed tomography using super-voxels and graph clustering", The Visual Computer, pp. 1-14, 2018.

[14] I. B. Păvăloiu, A. Vasilăţeanu, N. Goga, I. Marin, C. Ilie, A. Ungar, and I. Pătraşcu, "3D dental reconstruction from CBCT data", In: Proc. of IEEE 2014 International Symposium on Fundamentals of Electrical Engineering, pp. 1-6, 2014.

[15] Y. Fan, R. Beare, H. Matthews, P. Schneider, N. Kilpatrick, J. Clement, P. Claes, A. Penington, and C. Adamson, "Marker-based watershed transform method for fully automatic mandibular segmentation from CBCT images", Dentomaxillofacial Radiology, Vol. 48, No. 2, p. 20180261, 2019.

[16] S. Rueda, J. A. Gil, R. Pichery, and M. Alcañiz, "Automatic segmentation of jaw tissues in CT using active appearance models and semiautomatic landmarking", In: Proc. of 
International Conference on Medical Image Computing and Computer-Assisted Intervention, pp. 167-174, 2006.

[17] D. Kainmueller, H. Lamecker, H. Seim, M. Zinser, and S. Zachow, "Automatic extraction of mandibular nerve and bone from cone-beam CT data", In: Proc. of International Conference on Medical Image Computing and ComputerAssisted Intervention, pp. 76-83, 2009.

[18] F. Abdolali, R. A. Zoroofi, M. Abdolali, F. Yokota, Y. Otake, and Y. Sato, "Automatic segmentation of mandibular canal in cone beam CT images using conditional statistical shape model and fast marching", International Journal of Computer Assisted Radiology and Surgery, Vol. 12, No. 4, pp. 581-593, 2017.

[19] F. Gan, G. Ruan, and J. Mo, "Baseline correction by improved iterative polynomial fitting with automatic threshold", Chemometrics and Intelligent Laboratory Systems, Vol. 82, No. 1-2, pp. 59-65, 2006.

[20] D. York, "Least-squares fitting of a straight line", Canadian Journal of Physics, Vol. 44, No. 5, pp. 1079-1086, 1966.

[21] A. A. Taha and A. Hanbury, "Metrics for evaluating 3D medical image segmentation: analysis, selection, and tool", BMC Medical Imaging, Vol. 15, No. 1, p. 29, 2015.

[22] N. Otsu, "A threshold selection method from gray-level histograms", IEEE Transactions on Systems, Man, and Cybernetics, Vol. 9, No. 1, pp. 62-66, 1979.

[23] A. Z. Arifin and A. Asano, "Image segmentation by histogram thresholding using hierarchical cluster analysis", Pattern Recognition Letters, Vol. 27, No. 13, pp. 15151521, 2006.

[24] T. Kurita, N. Otsu, and N. Abdelmalek, "Maximum likelihood thresholding based on population mixture models", Pattern Recognition, Vol. 25, No. 10, pp. 1231-1240, 1992.

[25] D. J. Kroon and C. H. Slump, "MRI modalitiy transformation in demon registration", In: Proc. of IEEE International Symposium on Biomedical Imaging: From Nano to Macro, pp. 963-966, 2009.

[26] O. Ronneberger, P. Fischer, and T. Brox, "Unet: Convolutional networks for biomedical image segmentation", In: Proc. of International Conference on Medical Image Computing and
Computer-Assisted Intervention, pp. 234-241, 2015.

[27] Y. B. Chang, J. J. Xia, P. Yuan, T. H. Kuo, Z. Xiong, J. Gateno, and X. Zhou, "3D segmentation of maxilla in cone-beam computed tomography imaging using base invariant wavelet active shape model on customized two-manifold topology", Journal of X-ray Science and Technology, Vol. 21, No. 2, pp. 251-282, 2013.

[28] W. R. Crum, T. Hartkens and D. L. G. Hill, "Non-rigid image registration: theory and practice", The British journal of radiology, Vol. 77, No. suppl_2, pp. S140-S153, 2004.

[29] T. Vercauteren, X. Pennec, A. Perchant, and N. Ayache, "Diffeomorphic demons: Efficient non-parametric image registration", NeuroImage, Vol. 45, No. 1, pp. S61-S72, 2009.

[30] D. Shen, G. Wu, and H. I. Suk, "Deep learning in medical image analysis", Annual Review of Biomedical Engineering, Vol. 19, pp. 221-248, 2017.

[31] M. I. Razzak, S. Naz, and A. Zaib, "Deep learning for medical image processing: Overview, challenges and the future", In: Classification in BioApps, pp. 323-350, 2018. 